\title{
Data Fusion for Mobile Terminal Location
}

\author{
M. McGuire, K.N. Plataniotis, A.N. Venetsanopoulos \\ The Edward S. Rogers Sr. Department of Electrical and Computer Engineering, University of Toronto, \\ 10 King's College Road, Toronto, Ontario, M5S 3G4, Email: mmcguire@dsp.toronto.edu
}

\begin{abstract}
The location of mobile terminals in cellular networks is an important problem with applications in resource allocation, location sensitive browsing, and emergency communications. A key problem in mobile terminal location is the creation of a method that is robust to Non Line of Sight (NLOS) propagation, measurement quantization, and measurement noise and also has a reasonable implementation cost. This paper proposes a technique that fuses together location estimates obtained from propagation power loss measurements and propagation time measurements together to obtain a higher accuracy location estimate. The technique uses non-parametric estimation methods to estimate the covariance of each of the individual location errors to optimally fuse the location estimates together. It is shown how the data fusion location estimator is robust and has low implementation cost.
\end{abstract}

\section{INTRODUCTION}

Mobile terminal location is an important problem for modern cellular networks with applications to resource allocation, location-sensitive information browsing, and emergency communications. The characteristics of the radio channel between the mobile terminal and fixed location base stations are dependent on the location of the mobile terminal. This allows for the location of the mobile terminal to be estimated from measurements made of the radio propagation channels. The most popular proposed measurements are Received Signal Strength (RSS), Time of Arrival (ToA), and Time Difference of Arrival (TDoA) [1]. For RSS measurements, the signal power at the receiver is measured. For ToA measurements, the propagation times from multiple base stations to the mobile terminal is measured. For TDoA measurements, the differences between the propagation times to the mobile terminal for sets of two base stations are measured.

To obtain ToA measurements, the receivers need the times of transmission of the radio signals. To obtain this knowledge with the high accuracy is expensive [1]. RSS and TDoA measurements, however, can be obtained cheaply in digital cellular networks.

Accurate mobile terminal location estimation is trivial when the radio propagation is the shortest distance straight line paths between the mobile terminal and base stations is unobstructed, so called LOS propagation, and the measurement noise is negligible. In the urban areas of greatest interest to cellular network operators, Non Line of Sight (NLOS) propagation, when obstructions block the shortest distance propagation paths, is common. RSS and TDoA measurements made in real cellular networks are affected by noise.

The authors have previously demonstrated location estimation algorithms for both RSS and TDoA measurements that are robust to additive measurement noise and NLOS propagation [2], [3]. These algorithms are based on the use of non-parametric estimation techniques using survey data taken from the propagation environment.

This paper proposes data fusion to exploit the independence of the measurement noise between RSS and TDoA measurements to combine the measurements' respective estimates together to create a fused estimate with lower error. The robustness of this data fusion estimator is demonstrated.

In Section II, the signal model used to evaluate the location procedures is explained. A realistic simulated urban radio propagation environment with NLOS propagation is described. In Section III, the new estimation methods are outlined. Data fusion methods of the path loss and TDoA location estimates using on-line computation of the error covariances are shown. Section IV includes results that demonstrate that these methods are robust to quantization and variations in the measurement noise. Section $\mathrm{V}$ provides the conclusions of this work.

\section{SIGNAL MODEL}

The true location of the mobile terminal is denoted $\theta=$ $(x, y)$ where $(x, y)$ is the location of the mobile terminal. This paper will only consider two dimensional location but the methods are easily extended. For a given mobile terminal location $\theta$, the generated signal vectors are $(p, t, b)$ where $p$ is the vector of RSS path loss measurements, $t$ is the vector of TDoA measurements, and $b$ is a vector of the indices of the base stations that are the sources of measurements. Path loss measurements are obtained by subtracting the RSS measurements from the known transmit power in the decibel domain. Only measurements from the three base stations with the lowest path loss measurements taken out of the set of nine base stations closest to the mobile terminal are included in $(p, t, b)$ with the first entry of $b$ indicating the base station with the lowest path loss measurement. This truncation is a good approximation to actual field measurement practice.

We denote as $[x]_{k}$ the $k^{\text {th }}$ entry of vector $\boldsymbol{x}$. The unquantized measurement vectors for a mobile terminal at location $\theta$ are $\tilde{p}=\mathrm{p}(\theta, b)+\varepsilon_{p}$, and $\tilde{t}=\boldsymbol{G}\left(\mathrm{d}(\boldsymbol{\theta}, \boldsymbol{b})+\varepsilon_{\tau}\right) \cdot \mathrm{p}(\boldsymbol{\theta}, \boldsymbol{b})$ is a function giving the deterministic path loss values from the base stations indicated in $b$ such that $[p(\theta, b)]_{k}$ is the deterministic portion of the path loss from the base station indicated by $[b]_{k}$, and $\mathrm{d}(\theta, b)$ is a similar function giving the the lengths of the shortest propagation paths from the mobile terminal to the measuring base stations indicated in $b . \varepsilon_{p}$ and $\varepsilon_{t}$ are random variables representing measurement noise. $G$ is a difference matrix. In our simulations, TDoA measurements are calculated with respect to the time measurement for the base station $[b]_{1}$ : 


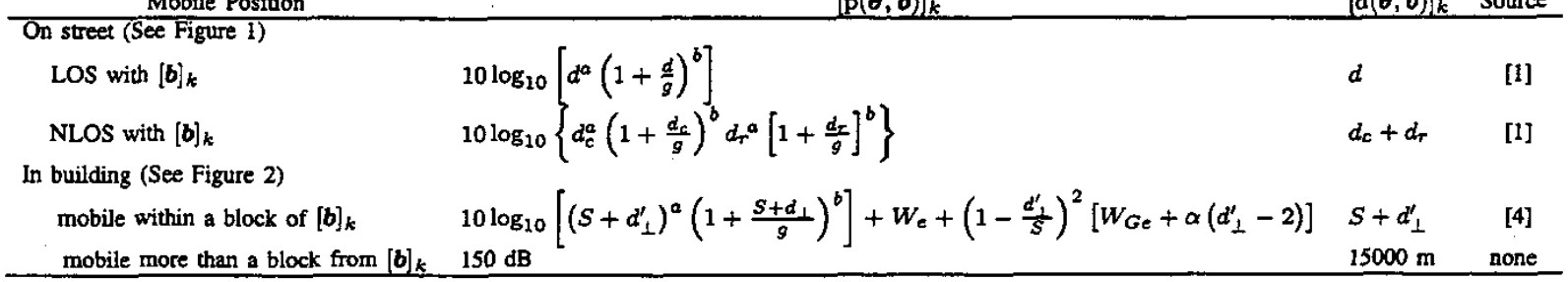

See associated figures for definitions of distances.

TABLE I

PROPAGATION MODELS FOR. SiMULATIONS $\boldsymbol{G}=\left[\begin{array}{ccc}1 & -1 & 0 \\ 1 & 0 & -1\end{array}\right]$. The simulated measurement error
values are independent between different base stations.

The simulated radio signal model used to evaluate the estimation algorithm is based on an urban radio propagation model used to evaluate other radio location methods with additional modeling information for propagation into buildings [1], [4]. The layout for a single base station's cell in this simulated urban environment is shown in Figure 1, where the shaded areas represent buildings.

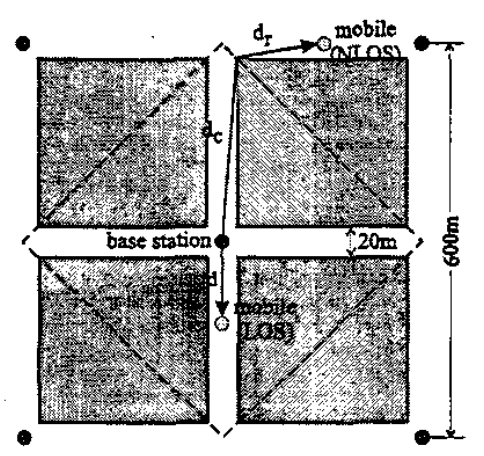

Fig. 1. Manhattan Propagation Model (Single Celi)

The calculation of $p(\theta, b)$ and $d(\theta, b)$ for a given location and base station in the simulated environment is given in Table I. For propagation to street locations the distances $d$, $d_{c}$, and $d_{r}$ are shown in Figure 1 . Distances $S, d_{\perp}$, and $d_{\perp}^{\prime}$ for NLOS propagation into a building are shown in Figure $\overrightarrow{2}$. The values for the other constants used in the propagation equations are listed in Table II. It is assumed that a base station cannot provide a high quality radio signal to distant mobile terminals located in buildings. The propagation model in Table I reflects this.

The random portion of the path loss for urban radio propagation, $\varepsilon_{p}$, has been shown to be well modeled as a Gaussian random variable [5]. In our simulations, we use a zero mean Gaussian random variable with standard deviation $\sigma_{p}$. The random portion of the propagation time measurements, $\varepsilon_{\tau}$, is the sum of two random processes: synchronization error, and excess delay caused by NLOS propagation from mobile

\begin{tabular}{|c|c|c|c|}
\hline Constant & Value & Constant & Value \\
\hline$a$ & 2.0 & $\alpha$ & $0.6 \mathrm{~dB} / \mathrm{m}$ \\
\hline$b$ & 2.0 & $\sigma_{p}$ & $2.0-8.0 \mathrm{~dB}$ \\
\hline & $150 \mathrm{~m}$ & $\sigma_{\tau}$ & $10.0-20.0 \mathrm{~m}$ \\
\hline$W_{e}$ & $10 \mathrm{~dB}$ & $q_{p}$ & $1.0 \mathrm{~dB}$ \\
\hline$W_{G e}$ & $20 \mathrm{~dB}$ & $q_{t}$ & $60.0 \mathrm{~m}$ \\
\hline \multicolumn{4}{|c|}{ TABLE II } \\
\hline
\end{tabular}

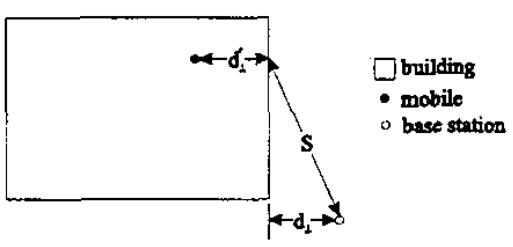

Fig. 2. Building Propagation Model

scatterers. Synchronization error for CDMA receivers, which are likely to be used in next generation cellular systems, has been shown to be well modeled as a Gaussian random variable [6], it is simulated as a zero mean Gaussian random variable with a standard deviation $\sigma_{\tau}$. The simulated synchronization noise variance is the same for all base stations, justified by simulations studies that show the synchronization variance is nearly constant for a large range of receiver signal to noise ratios in urban micro-cell propagation [1]. We simulate the effects of NLOS on the time measurements as an exponential random variable with a mean value of $\lambda[7]$.

The measurements are quantized making the observed measurement vectors: $\boldsymbol{p}=\operatorname{mnd}\left(\tilde{\boldsymbol{p}} / q_{p}\right) q_{p}$, and $\boldsymbol{t}=\operatorname{rnd}\left(\tilde{\boldsymbol{t}} / q_{t}\right) q_{t}$. The constants $q_{p}$ and $q_{t}$ are the quantization constants, and $y=\operatorname{rnd}(x)$ is a vector function such that $[y]_{k}$ is the closest integer to $[x]_{k}$. The quantization in the simulations are listed in Table II.

Of primary consideration for the application of data fusion of RSS path loss and TDOA measurements is the independence of $\varepsilon_{p}$ and $\varepsilon_{\tau}$. If these vectors are perfectly dependent then data fusion of RSS and TDoA would give no improvement in accuracy as both measurements contain identical information. A radio propagation path loss measurement is determined by 
the sum of the received signal power over all propagation paths between a base station and the mobile terminal. A TDoA measurement is determined by the propagation delays of the shortest propagation paths from the involved base stations to the mobile terminal. Discrepancies between the aspects of propagation engaged in each measurement type ensure there is some independence of the measurement noise for each measurement type.

\section{ESTIMATION METHODS}

The main focus of this paper is on data fusion of estimates of mobile terminal location from path loss and TDoA measurements. Estimation methods for calculating estimates of mobile terminal location using either path loss measurements or TDoA measurements alone is a fairly mature field. In this paper, we use single measurement types estimates calculated using non-parametric techniques, survey data, and prior knowledge of mobile terminal location available from the hand off algorithm. These methods will only be briefly described here since a full analysis has been performed in the literature [2], [3].

The single measurement estimation method described here are based upon the existence of survey data for the radio propagation environment. The survey data of consists of a set of locations with three measurement vectors for each location. The location of the $j^{\text {th }}$ survey point is denoted $\boldsymbol{\theta}_{j}$. The measurement vectors of survey point $j$ are $\left(b_{j}, p_{j}, t_{j}\right) . b_{j}$ is a vector of base station indices which indicates which base stations made the measurements at the $j^{\text {th }}$ survey point. $\boldsymbol{p}_{j}$ is the vector of path loss measurements for the $j^{\text {th }}$ survey point. $t_{j}$ is the TDoA measurement vector for the $j^{\text {th }}$ survey point. The measurement process for the survey points are identical to measurement process used for the location measurement $(b, p, t)$ described in Section $I$. In the cell for each base station are located $N=500$ survey points. The locations of these survey points are uniformly distributed over the cell area. Techniques on estimation of methods for bounding the number of required survey points is presented in [2].

The first step of non-parametric location estimation algorithms is to use the base station index vector, $b$, to identify the general area in which the mobile terminal is likely to be located. Using the measurement protocol defined in Section II, $[b]_{1}$ identifies the base station with the lowest path loss measurement to the mobile terminal. We assume that the mobile terminal is located either in the cell associated with base station $[b]_{1}$ or in one of the cells adjacent to it. Using this prior location assumption, we collect the survey data for all survey points located in the cell for base station $[b]_{1}$ and the immediately adjacent cells where $b_{j}$ matches $b$ are kept for the location algorithm.

The single measurement type estimation equation, where $z$ is the measurement type, $z$ is the measurement vector, and $\boldsymbol{z}_{j}$ is the measurement vector for survey point $j$, is $\hat{\theta}_{z}=$ $\sum_{j=1}^{n} \theta_{j} w_{j}(z)$ with $w_{j}(z)=\mathrm{K}_{z}\left(z-z_{j}\right) /\left(\sum_{k=1}^{n} \mathrm{~K}_{z}(z-\right.$ $\left.\left.z_{k}\right)\right) . K_{z}(\cdot)$ is called the kernel function for measurement type $z$. $z$ is replaced by $p$ for path loss measurements and $t$ for
TDoA measurements with similar substitutions for $z$ and $z_{j}^{\prime}$. It has been previously shown that a Gaussian probability density function is a good choice for the kernel function:

$$
\mathrm{K}_{z}(x)=\frac{1}{(2 \pi)^{\frac{m_{z}}{2}}\left|C_{z}\right|^{\frac{1}{2}}} \exp \left(-\frac{x^{T} C_{z}^{-1} x}{2}\right) .
$$

The constants are $m_{p}=3$ and $m_{t}=2$. The covariance matrices are $C_{p}=h_{p}^{2} \sigma_{p}^{2} I_{3}$, where $I_{m}$ is an $(m \times m)$ identity matrix, and $\boldsymbol{C}_{t}=h_{t}{ }^{2} \sigma_{\tau}{ }^{2} \boldsymbol{G} G^{T}$. The constants $h_{p}$ and $h_{t}$ are defined as the kernel width constants. The effects of varying values of the constants will examined in Section IV where it will be shown that the estimators are robust to variations of the constants' values.

Using the kemel functions above and the survey points, estimates of the covariances for the estimate errors are calculated using $R_{z}=\left(\sum_{j=1}^{n} \theta_{j} \theta_{j}^{T} w_{j}(z)\right)-\hat{\theta}_{z} \hat{\theta}_{z}^{T}$.

We now discuss data fusion of $\hat{\theta}_{p}$ and $\hat{\theta}_{t}$ to obtain a lower error estimate $\hat{\theta}$. We propose the application of a standard approach in data fusion of linearly combining the estimated values of mobile terminal location obtained from different measurements to this problem. We define the dual estimate vector as $\hat{\theta}_{k}=\left[\hat{\theta}_{p}^{T} \hat{\theta}_{t}^{T}\right]^{T}$ and the dual location vector as $\theta_{k}=\left[\theta^{T} \theta^{T}\right]^{T}$. The estimate fusion calculation is given by $\hat{\theta}=W \hat{\theta}_{k}$ where $W$ is a weight matrix. The optimal weight for each component estimate is based on its covariance with an estimate given a higher weight if its covariance is low. The optimal $\boldsymbol{W}$, which gives the lowest fused covariance is given by [8]:

$$
\boldsymbol{W}=C_{k}^{-1} A^{T}\left(A C_{k}^{-1} A^{T}\right)^{-1}
$$

with

$C_{k}=\mathrm{E}\left\{\left[\boldsymbol{\theta}_{k}-\hat{\boldsymbol{\theta}}_{k}\right]\left[\boldsymbol{\theta}_{k}-\hat{\boldsymbol{\theta}}_{k}\right]^{T} \mid \boldsymbol{p}, t\right\}=\left[\begin{array}{cc}\boldsymbol{R}_{p} & \boldsymbol{R}_{p t} \\ \boldsymbol{R}_{p t}{ }^{T} & \boldsymbol{R}_{t}\end{array}\right]$

and $\boldsymbol{A}=\left[\begin{array}{ll}\boldsymbol{I}_{2} & \boldsymbol{I}_{2}\end{array}\right] . \boldsymbol{R}_{p t}$ is the cross covariance of the path loss and TDoA measurement estimates. This method is robust in that linear combination using $W$ calculated using (2) with the true covariance matrices will always result in a fused estimator with lower covariance than the individual estimators.

In practice, the covariances $\boldsymbol{R}_{p}, \boldsymbol{R}_{t}$ and $\boldsymbol{R}_{p t}$ can only be estimated and the effectiveness of this approach in reducing the covariance of the final estimate is dependent on the accuracy of the covariance estimates. Also, to calculate $\boldsymbol{R}_{p t}$, the estimators for each measurement type cannot be run in parallel as they must exchange information to calculate the cross covariance matrix estimate. For the results on estimate fusion, we explore parallel estimation, and thus assume that the location estimate error for each measurement type is independent in the data fusion calculation. To this end, the covariance matrix in the weight calculation (2) is calculated using the assumption $\boldsymbol{R}_{p t}=\mathbf{0}$.

The main advantage of parallel estimation for each measurement type is the independence of the data fusion method from the location estimate calculation for each measurement type. The estimation calculations can be replaced with another lo- 
cation estimate calculation with an associated error covariance estimate and the data fusion technique can still be applied.

\section{RESULTS}

The estimators are evaluated in the simulated urban environment described in Section II. For each single set of parameter values, we simulate 10000 Monte Carlo trials with a new survey set for every 100 trials. The true mobile terminal position is sampled from a uniform distribution over a single cell. The figure of merit used to evaluate the location estimators is the Root Mean Square Error (RMSE), the square root of mean squared distance from the tnue mobile terminal location and the estimated location.

The first set of simulation are performed to calculate the optimal values of the kernel width constants $h_{t}$ and $h_{p}$ and to test estimator performance if sub-optimal values are used. The result is shown in the contour plot in Figure 3 of the RMSE for different kernel width constants for specific values of measurement noise standard deviations. The plot shows that data fusion methods is robust to variations of the kernel width parameters with a large region in the plots having RMSE values almost as low as the to the optimal value. The optimal kernel width values are found to be $h_{p}=1.2$ and $h_{t}=1.0$.

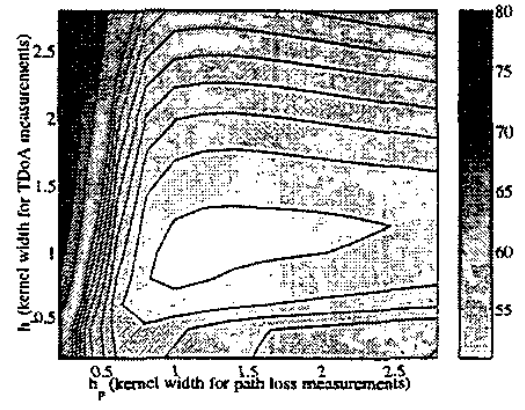

Fig. 3. Kernel Widths for Estimate Fusion $\left(\sigma_{p}=6 \mathrm{~dB}, \sigma_{\tau}=15.0 \mathrm{~m}\right)$

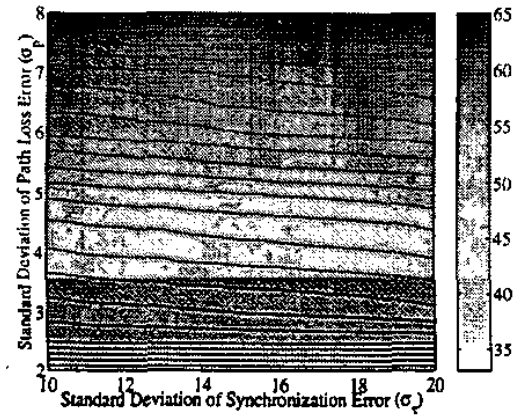

Fig. 4. RMSE of Best of RSS and TDOA Estimators $\left(h_{p}=1.2\right.$ and $h_{t}=$ 1.0)

The next set of simulations were performed to find the performance of location estimators using only one measurement type. The optimal kemel width values described above are used. A contour plot of the lowest RMSE of either the path loss or TDoA location estimator for a range of measurement noise standard deviations is shown in Figure 4. Note that the estimators give good performance even with large magnitude measurement noise and despite quantization.

The improvement of the data fusion estimators over estimators using only one single measurement type are demonstrated in the next set of simulation results. For fixed values of $\sigma_{p}$ and $\sigma_{\tau}$, the RMSE of the results for the data fusion method is subtracted from the lowest RMSE of the estimators using either path loss or TDOA measurements only. The resulting value is the improvement of the data fusion method over estimation using a single measurement type. Figure 5 shows the improvement of the data fusion method.

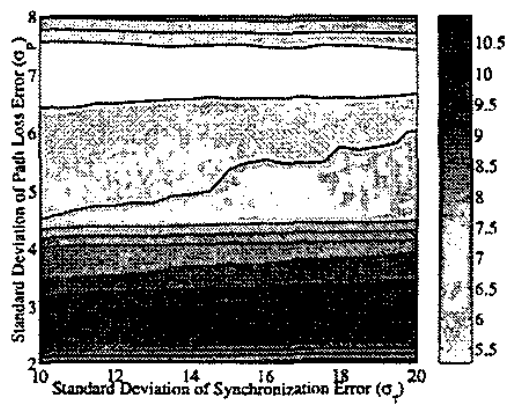

Fig. 5. RMSE Improvement for Data Fusion $\left(h_{p}=1.2, h_{t}=1.0\right)$

\section{CONCLUSIONS}

This paper demonstrates a method of data fusion for path loss and TDoA measurements. It was shown that this method improves location accuracy, is robust to different levels of measurement noise, and has low computational cost.

\section{ACKNOWLEDGMENTS}

This work was supported by the Nortel Institute for Telecommunications

\section{REFERENCES}

[1] J. Caffery, Jr and G. Stuber, "Subseriber location in CDMA cellular networks," IEEE Transactions on Vehicular Technology. vol. 47, no. 2 , pp. 406-416. May 1998.

[2] M. McGuire, K. Plataniotis, and A. Venetsanopoulos, "Location of mobile terminals using time measurements and survey points." March 2003 , accepted by IEEE Transactions on Vehicular Technology.

[3] M. McGuire and K. Platariotis, "Estimating position of mobile terminal from path loss measurements with survey data," Wireless Communications and Mobile Computing, vol. 3, no. 1, pp. 51-62, February 2003.

[4] J.-E. Berg, "Building penetration loss along urban street microcells," in Personal, Indoor and Mobile Radio Communication Conference, PIMRC, October 1996, pp. 795-797.

[5] J. Parsons, The Mobile Radio Propagation Channel, 2nd ed. Toronto, Ontario: John Wiley \& Sons, Inc., 2000.

[6] H. Gombachika and O. Tonguz, "Influence of multipath fading and mobile unit velocity on the performance of PN tracking in CDMA systems," in IEEE Vehicular Technology Conference, May 1997, pp. 2206-2209.

[7] P.-C. Chen. "A cellular based mobile location tracking system," in IEEE Vehicular Technology Conference, May 1999, pp. 1979-1983.

[8] Y. Zhu, Multisensor Decision and Estimation Fusion. Boston: Kluwer Academic Publishers, 2003. 